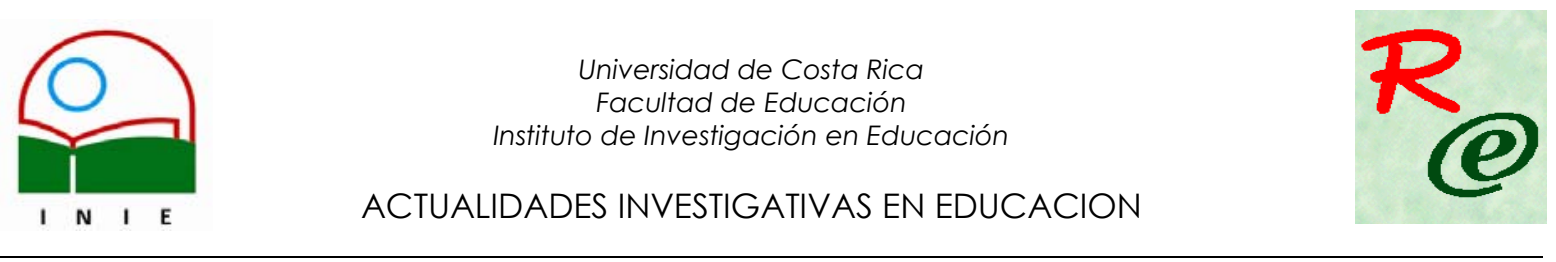

\title{
HACIA LA REDEFINICIÓN DEL CRÉDITO ACADÉMICO EN LA UNIVERSIDAD DE COSTA RICA
}

\author{
Ana Isabel Mora Vargas ${ }^{1}$ \\ Isabel Gallardo Alvarez ${ }^{2}$
}

Resumen: El propósito de este artículo es ofrecer un primer avance del proyecto de investigación inscrito en el Instituto de Investigaciones en Educación (INIE) acerca de la reconceptualización del crédito académico, entendido como la unidad valorativa de la carga académica del estudiante de la Universidad de Costa Rica. Se incluye información tanto de los antecedentes del concepto créditos en la educación superior pública costarricense como los planteamientos internacionales del proyecto Tunning que pretende mejorar la calidad de la educación superior en Europa. Se ofrece también a manera de consideraciones finales, algunas problemáticas sobre el cálculo de créditos en los cursos de los planes de estudios en la Universidad de Costa Rica.

Palabras claves: CRÉDITO/ CRÉDITO ACADÉMICO/ CARGA ACADÉMICA ESTUDIANTIL/ UNIDAD VALORATIVA/ TRABAJO ESTUDIANTIL/ CURSO/ PLANIFICACIÓN CURRICULAR/ PLAN DE ESTUDIO/ EDUCACIÓN SUPERIOR COSTARRICENSE/

Abstract: The purpose of this article is to offer a first advance of the research project ascribed to the Institute of Educational Research dealing with the re-conceptualization of the academic credit as a unit to assess the academic load of students at the Universidad de Costa Rica. The piece includes information on the background of the concept of credit in the Costa Rican improve the quality of higher education. As a conclusion the article reviews a recent survey on the problems to calculate credits in the courses of study plan of Universidad de Costa Rica.

Keywords: CREDIT/ ACADEMIC CREDIT/ STUDENT ACADEMIC LOAD/ ASSESSMENT UNIT/ STUDENT WORK COURSE/ CURRICULAR PLANNING/ STUDY PLAN/ COSTA RICAN HIGHER EDUCATION/

\section{Introducción}

¿Refleja el crédito la carga académica estudiantil? Para responder a esta pregunta se ha inscrito un proyecto en el Instituto de Investigación en Educación (INIE), de la Facultad de Educación de la Universidad de Costa Rica, titulado "Hacia la redefinición del crédito: una unidad valorativa de la carga académica del estudiante en la educación superior" ( $N^{\circ} 724-$ A2-172) que se propone diagnosticar el concepto de crédito y sus implicaciones en la planificación curricular y en el esfuerzo del trabajo estudiantil.

\footnotetext{
${ }^{1}$ Magister Scientiae en Educación con énfasis en Investigación Educativa. Licenciada en Ciencias de la Educación con énfasis en Currículo. Bachiller en Enseñanza Especial con énfasis en Trastornos de la Comunicación. Profesora de la Escuela de Formación Docente de la Facultad de Educación de la Universidad de Costa Rica. Investigadora del Instituto de Investigación en Educación (INIE). Miembro de la Comisión de Investigación y Trabajos Finales de Graduación. Correo electrónico: aimora@cariari.ucr.ac.cr

${ }^{2}$ Licenciada en Filología Española, Bachillerato en la Enseñanza del Castellano y la Literatura ambos de la Universidad de Costa Rica. Profesora de la Escuela de Formación Docente. Investigadora del Instituto de Investigación en Educación (INIE). Directora y asesora de diferentes Trabajos Finales de Graduación.

Correo electrónico: isabella gallardo@hotmail.com
}

Artículo recibido: 21 de octubre, 2005

Aprobado: 12 de diciembre, 2005 
La razón fundamental para inscribir un proyecto de este tipo obedece a la necesidad de redefinir esta concepción, dado que el crédito, como unidad valorativa de la carga académica estudiantil, se establece en forma oficial en la Universidad de Costa Rica en 1975 y no ha variado desde entonces, mientras que las condiciones del estudiante universitario para organizar su carga académica y adquirir y procesar la información sí lo han hecho. Esto hace indispensable que el concepto de crédito se ajuste también al cambio de los tiempos.

Como un primer paso para elaborar la investigación, se consideró necesario conocer los antecedentes históricos de la definición del crédito, así como la función que este ha ido adquiriendo dentro de la vida universitaria. Por eso se pretende buscar en documentos del CONARE, del Consejo Universitario y en otros, la evolución histórica de este concepto, así como la normativa que se utilizó para fijarlo en las universidades. También se cree importante conocer cómo se ha trabajado sobre este concepto en otras latitudes y una forma es indagando sobre lo que se trabaja actualmente en Europa, en el marco del Proyecto Tunning que se está ocupando de redefinir no solo el concepto crédito, sino también la calidad de la educación superior. Este proyecto europeo además, está teniendo repercusiones en América Latina y ya se están viendo algunos frutos en México, donde se está revisando también la educación superior.

Este artículo quiere ofrecer información acerca de los antecedentes de la definición del crédito, la normativa para la aplicación del mismo en los planes de estudio de las carreras de la educación superior pública costarricense. Así mismo pretende señalar algunos de los cambios que en este momento se están haciendo en Europa sobre el tema, que pareciera van a impactar la educación de América Latina en general y de Costa Rica en particular.

Como consideraciones finales del artículo, se ofrecen los resultados más relevantes de un sondeo realizado durante el primer ciclo del 2005, en las unidades académicas de la Universidad de Costa Rica que se seleccionaron para el estudio. De este sondeo se consiguió la información pertinente para elaborar un instrumento que permitirá diagnosticar la situación actual del crédito en la universidad y así obtener elementos para su posterior reconceptualizacion.

El proyecto inscrito, no solo busca la reconceptualización del concepto crédito sino que va más allá e incluye una etapa de plan piloto con las unidades académicas, para 
aplicar el nuevo concepto enunciado de crédito en los planes de estudios. Los resultados de este proceso se comunicarán en su momento.

El artículo que se presenta a continuación, está organizado en cuatro apartados: el primero de ellos proporciona una somera revisión de los antecedentes históricos de cómo fue adoptado el crédito como unidad valorativa del trabajo académico del estudiante en la educación superior costarricense; el segundo reseña las actividades que CONARE en su época (1977) propuso para que la adaptación del crédito fuera semejante en las universidades públicas del país así como algunos de los problemas que se vivieron en su aplicación. El tercero se refiere a la situación que actualmente atraviesa este tema así como las diversas funciones que cumple dentro de la vida universitaria. En el último apartado hace un recuento del Proyecto Tuning, dando énfasis a la parte que estudia el tema del crédito y la búsqueda que las universidades europeas han emprendido para que todos los centros de educación superior adopten el mismo criterio sobre el tema.

Finalmente, se ofrece a manera de consideraciones finales, obtenidas en un sondeo reciente acerca de los problemas que presenta el cálculo de créditos en los cursos de los planes de estudios en la Universidad de Costa Rica

\section{Antecedentes}

La necesidad de contar con una unidad que permita valorar y medir el trabajo del estudiante, se presentó en la educación superior costarricense desde 1927. Pero, no es sino hasta la sesión $N^{\circ} 50$ del Consejo Universitario de la Universidad de Costa Rica de 1952, donde se retoma el tema y se lleva al tapete de discusión el hecho de que la Escuela de Agronomía empleaba un concepto de crédito para evaluar las diferentes materias. Esta Escuela utilizó, en un principio, el criterio de la importancia de los cursos y posteriormente el de horas cursadas para conferírselo a cada una de sus materias. Al respecto, se tomó como base el criterio de otorgar un crédito por una hora de clase teórica y en las lecciones prácticas media hora semestral. En la escuela citada el estudiantado debía cumplir con un cierto número de créditos para graduarse. El sistema utilizado por la Escuela de Agronomía no contaba, en ese entonces, con la aprobación del Consejo Universitario (Acta $N^{0} 50$ del Consejo Universitario, 1952). 
En el año 1952, la Escuela de Pedagogía, hoy Facultad de Educación de la Universidad de Costa Rica, presentó al Consejo Universitario un plan de creditaje para valorar las carreras de dicha Escuela, el cual fue enviado a la Comisión de Planes y Programas de Estudios donde se determinó que "no puede establecerse un sistema de créditos en una escuela, sin antes haber fijado una norma general para toda la universidad" (Sesión 50 C.U., 1952).

Al respecto, el Consejo Universitario integra una comisión con el propósito de que estudie y presente una reglamentación acerca del sistema de otorgamiento de créditos que permita la validación en instituciones del extranjero, mientras tanto se suspende el sistema de aplicación de créditos existente en la Escuela de Agronomía.

El crédito se establece, en forma oficial, el 4 de julio de 1953 para todas las escuelas de la Universidad de Costa Rica. En los Estatutos Orgánicos de 1956, de 1962 y de 1968 (artículo 103), se incluyen los criterios de la aplicación del crédito.

El crédito como medida académica fue usado únicamente por la Universidad de Costa Rica hasta 1975. El concepto utilizado hacía equivaler un crédito por cada hora de lección teórica en el horario formal y medio crédito por cada hora de práctica o laboratorio (CONARE, 1976).

Hasta esta fecha, la Universidad de Costa Rica, como única universidad existente en el país, utiliza un sistema de crédito, el cual no ofrece ningún problema (CONARE, 1976). A partir de este año, con la creación de otras instituciones de educación superior estatal: el Instituto Tecnológico de Costa Rica (1970), la Universidad Nacional (1973) y la Universidad Estatal a Distancia (1975), se siente la necesidad de unificar la definición de crédito en la educación superior. Varias razones fundamentaron la creación de un convenio entre ellas: la necesidad de coordinar el sistema de otorgamiento de grados y títulos en la educación superior, la necesidad de facilitar el reconocimiento de estudios, grados y títulos y la transferencia de estudiantes entre las instituciones. Otra razón que tuvo importancia fue la de contar con una unidad de medida para la actividad académica del estudiante, que fuera común entre las universidades estatales. 
Por consiguiente, en 1976 se aprueba oficialmente, para las cuatro instituciones de educación superior existentes, una definición oficial del crédito (CONARE, 1976). Desde entonces el concepto de crédito no ha variado y es oficial para todas las universidades estatales. Se define como la

... unidad valorativa del trabajo del estudiante, que equivale a tres horas reloj de trabajo semanal durante 15 semanas, aplicadas a una actividad que ha sido supervisada, evaluada y aprobada por el profesor. (CONARE 1977).

Esta definición de crédito se refiere a la carga académica estudiantil de tiempo completo, definiéndose al estudiante de tiempo completo, como aquel que matricula una carga académica de 18 créditos por semestre y dedica, a las actividades académicas, un promedio de 54 horas a la semana. CONARE es quien fija que para medir la carga académica del estudiante, no solo se tome en cuenta los créditos sino también si el alumno dedica su tiempo completo al estudio, por eso es que también se preocupa por fijar como se debe medir el tiempo completo de dedicación al estudio (CONARE.1977)

El criterio para distribuir dieciocho créditos por ciclo, corresponde a la normativa emitida por CONARE, en relación con el grado, ya sea Bachillerato o Licenciatura. En la práctica, las Unidades Académicas organizan los cursos por ciclo, agrupando entre cuatro o cinco por semestre y los créditos se acomodan alrededor de dieciocho, quedando en algunos ciclos una cantidad de créditos ligeramente superior o inferior al número solicitado.

El crédito se considera como una unidad de medida que debe reflejar el esfuerzo académico estudiantil y el grado en que la institución contribuye para que este complete su formación. El Consejo Nacional de Rectores (CONARE), en 1976, ofrece una guía a los encargados de la planificación educativa y curricular, para orientar la tarea de asignar adecuadamente los créditos de las correspondientes asignaturas o actividades de un plan de estudios. Se destaca la importancia del tiempo que el estudiante utiliza para la promoción de una asignatura, el área de estudio y el tipo de metodología (CONARE, 1976).

A partir de 1988, con el Seminario sobre la asignación de créditos convocado por CONARE, el tema del crédito ha sido muy discutido en seminarios y congresos; donde se señalan los problemas que presenta la asignación de créditos a los cursos y las distintas interpretaciones del concepto en cada institución universitaria estatal. 


\section{Documento de CONARE para la aplicación de la nueva definición de crédito (1977)}

Después de que CONARE llega a una definición de crédito en 1975, esta institución publica un documento, cuyo objetivo es ayudar a las diferentes universidades públicas a poner en práctica esta definición. El texto titulado "Nomenclatura de grados y títulos en la Educación Superior" de CONARE (1977), recoge normativa sobre el crédito que hasta la fecha ha venido consultándose y aplicando en la Educación Superior, puesto que no ha habido ningún cambio desde entonces.

En el capítulo tres, titulado "Algunas definiciones adoptadas para uso común en la educación superior" se consigna la definición de "crédito" y se dice que este se usará en la Educación Superior para "designar a unidades valorativas de la actividad académica del estudiante" (CONARE, 1977, p. III-2) y recuerda que la definición que se había usado hasta ese momento, era aquella que indicaba que el crédito equivalía "a una hora de lección teórica formal o a dos horas de práctica o laboratorio, también en horario formal' (CONARE, 1977, p. III-2.)

Esta nueva definición de crédito buscaba incluir toda la actividad académica del estudiante a saber: horas asignadas en el horario formal, horas de estudio individual, así como la práctica adicional de acuerdo con un criterio subjetivo de estimación.

Este último aspecto mencionado obliga a tomar en cuenta ciertas características del estudiante que pueden afectar este tiempo de estudio adicional. Las características contempladas en este documento son: capacidad intelectual, aptitud para el estudio y condición socioeconómica, entre otras, que definían a lo que se consideró el estudiante promedio. Se consideró que este era aquel que se encuentra situado "en la media en aspectos como los citados anteriormente (capacidad intelectual, aptitud para el estudio y condición socioeconómica)" mediante el rendimiento académico. (CONARE, 1977, p. III-5)

Este rendimiento académico se relaciona con la carga académica de un estudiante promedio, esta deberá ser de 54 horas semanales, "lo que supone una dedicación diaria de nueve horas por cinco días a la semana, más cinco horas el sábado y cuatro el domingo" (CONARE, 1977, p. III-6). Así se puede decir que el estudiante promedio de tiempo 
completo "es aquel que cursa 18 créditos que equivalen 54 horas de trabajo semanal" (CONARE, 1977, p. III-6).

En este texto del CONARE se proporciona una guía para asignar créditos de acuerdo con la nueva definición y con la caracterización del estudiante promedio. Esta guía se encuentra consignada en el capítulo cuarto que es, como su nombre los indica, una guía para que las diferentes unidades académicas, programas y cursos pasen a asignar créditos a sus asignaturas basados en la definición, aprobada por CONARE en 1976.

En esta guía se advierte que existen ciertas actividades universitarias a las que no se les asigna créditos. Entre ellas se encuentra la actividad deportiva y la cultural (esta última actualmente sí tiene asignados créditos en la Universidad de Costa Rica) así como los proyectos de graduación y los trabajos comunales.

Este texto del CONARE consigna los resultados de una investigación que se hizo a una muestra de algunas carreras de todas las áreas del conocimiento, tanto de la Universidad de Costa Rica, como de la Universidad Nacional y del Instituto Tecnológico, donde se estudió la aplicación de la definición de crédito, tomando en cuenta dos aspectos: el primero fue la aplicación de la definición de crédito de igual forma a todas las áreas del conocimiento y el segundo, se relaciona con los aspectos que deben considerarse, en forma individual, para cada área y para cada tipo de metodología por las actividades que tiene que realizar el estudiante en el curso. Uno de los resultados interesante a que se llegó con este estudio, fue el referente al tiempo real que el estudiante promedio dedica al estudio.

En cuanto al número de horas semanales, se especificó que un estudiante de tiempo completo tenía que dedicar al estudio era de 54, pero estas fueron sobrepasadas en las tres instituciones:

$\checkmark$ En la Universidad de Costa Rica: los estudiantes dedican a sus estudios entre 29 y 67 horas semanales

$\checkmark$ En el Instituto Tecnológico: los estudiantes estudian entre 53 y 84 horas semanales

$\checkmark$ En la Universidad Nacional: dedican entre 31 y 66 horas semanales.

El mínimo de horas que se observa en los resultados fue obtenido únicamente en tres carreras, mientras que el máximo se aplicaba para la mayoría de las carreras investigadas. 
Estos resultados muestran que hay un exceso en la carga de horario del estudiante y esta variabilidad amerita una revisión de las horas asignadas a los cursos, con el fin de determinar si lo estipulado responde a la realidad. Con base en lo anterior, los investigadores de CONARE concluyeron que el exceso de horas se debe, sobre todo, a "una sobrestimación de las horas de estudio independiente necesarias para la promoción del curso" (CONARE, 1977, p. IV-32). Agregan que esta sobrestimación pareciera que se relaciona con el sistema de pedir por separado, a cada profesor, que estime el número de horas destinado al estudio de su materia, pues como seres humanos cada profesor le da más importancia a su materia y en ocasiones su apreciación supera el tiempo real.

De acuerdo con estos resultado se recomienda en el documento de CONARE que para la asignación de créditos "no debe intervenir una sola persona sino que debe ser revisado en distintas etapas y por grupos, en los cuales se emitan y compatibilicen los distintos criterios que se pueden dar" (CONARE, 1977, p. IV-36).

Otro aspecto al que se refiere el texto del CONARE es la dificultad que conlleva la transición de un sistema de asignación de créditos a otro diferente.

Este problema lo sufrió, sobre todo la Universidad de Costa Rica por ser la institución que tenía consolidada una forma de asignar créditos a las asignaturas. Esta transición la afectó en dos aspectos, en el número de créditos que ya habían ganado los estudiantes y las medidas que había que adoptar para reconocerlos y adaptarlos a la nueva definición sin afectar al estudiante, sobre todo en el aspecto financiero, pues en ese entonces se tenía la idea que lo que el alumno pagaba era la hora de clase recibida sin tomar en cuenta otros beneficios que la Universidad daba a los estudiantes como eran las tutorías, el servicio de biblioteca, los laboratorios y el uso de servicios, entre otros, que con esta forma de concebirlo si se tomaban en cuenta para calcular esta nueva definición costo económico de cada crédito.

Para adoptar esta definición de crédito, según el CONARE, era necesario seguir un proceso, en el que se involucraran una gran cantidad de personas de las Universidades, porque no se trataba de un proceso individual, sino más bien institucional. En este documento que se comenta, CONARE consigna seis pasos que las Universidades debían seguir para asignar créditos a las asignaturas. Los pasos incluían a la Vicerrectoría de 
Docencia, a las unidades académicas, a los profesores que laboraban en cada una de las unidades académicas, al director de cada una de las unidades académicas, a la Oficina de Planificación y finalmente, al Consejo Nacional de Rectores. Además se incluían procesos que implicaban trabajo individual y compartido de profesores, así como comunicación entre la unidad académica, la vicerrectoría y formación de comisiones ad-hoc, si las partes no estaban de acuerdo con la asignación de créditos a las distintas asignaturas del plan de estudio.

Al seguir los procedimientos estipulados, cada unidad académica podía tener la certeza que la asignación de créditos iba a ser satisfactoria e iba a beneficiar a los estudiantes de las distintas universidades públicas.

Uno de los intereses por los que se propuso en 1975, esta nueva definición de crédito era buscar la excelencia académica entre los estudiantes. Se considera que la excelencia académica que alcanza un estudiante es un concepto fundamental para definir los distintos aspectos de la vida universitaria. Al definir este concepto de excelencia académica se observa que no existe una sino varias definiciones. Para algunos profesores entrevistados por CONARE, el concepto es "el contraste entre fines y logros; es el grado de consecución de los objetivos de la educación" (CONARE, 1977, p. V-5). Otros consideran que la "Excelencia académica es la eficiencia de un Sistema de Educación Superior, desde el punto de vista académico" (CONARE, 1977, p. V-5. y finalmente, otros opinaron que es "la capacidad para investigar; la excelencia estriba en la originalidad de la investigación que un egresado del sistema puede producir" (CONARE, 1977, p. V-5).

La lectura de estos tres conceptos muestra que no hay un nivel óptimo de excelencia, sino distintos grados, es una noción cambiante y dinámica, por lo que es difícil de medir. Para llegar a una mejor conceptualización de excelencia académica se incluyeron distintas variables que influyen en su consecución, con el agregado que estas variables si se pueden medir. Estas se clasifican en aquellas que son relativas al profesor, las que son inherentes al estudiante, a los recursos educativos y otras. Se pueden enumerar de la siguiente forma:

1. Relativos al profesor

- Estudiantes por profesor

○ Carga docente 

- Dedicación a la enseñanza
- Formación (académica y docente)
o Experiencia y estabilidad en el cargo

2. Inherentes al estudiante
- Formación previa a la Educación Superior
o Tiempo de dedicación al estudio

3. Recursos educativos
- Disponibilidad de libros, revistas, microfilms, facilidades audiovisuales
- Equipo
- Planta física
- Apoyo administrativo

\section{Otros:}
- Estructura de los planes de estudio
- Contenido de los planes de estudio
- Adecuación de los planes a la realidad del país
- Calidad del personal docente
- Relaciones humanas entre profesor-estudiante
- Calidad del estudiante
- Calidad del personal docente
- Metodología de enseñanza
- Limitaciones económicas del estudiante

Todo lo enumerado anteriormente se puede medir, evaluar. Una vez evaluado es posible establecer la excelencia académica que puede llegar a tener un estudiante e incluir estos resultados, como una variable más para asignarle un creditaje a una asignatura específica.

Los conceptos hasta aquí enunciados muestran algunos de los pasos que se siguieron en 1975 para poner en funcionamiento en las universidades públicas, lo que en aquel entonces se consideraba una nueva definición de crédito que era necesario hacer llegar a los cursos e integrar a la vida universitaria. 


\section{Situación actual del crédito}

En 1988, CONARE organiza un seminario sobre créditos con la participación de las cuatro universidades públicas, en él se señalan los problemas más relevantes para el cálculo de créditos (Seminario sobre el crédito, CONARE, 1988). En 1990 fue un tema polémico en el Quinto Congreso Universitario y, en 1992 en los acuerdos del Quinto Congreso Universitario, la resolución $N^{\circ} 15$ se refiere a la necesidad de revisar la operacionalización del crédito como unidad valorativa del trabajo estudiantil. Finalmente en el año 2005, a petición de los vicerrectores de docencia de las distintas universidades públicas del país se creó una comisión interinstitucional y uno de sus objetivos es el de estudiar la aplicación del concepto de crédito

En el Seminario sobre el crédito, participaron las cuatro universidades estatales: Universidad de Costa Rica (UCR), Universidad Nacional (UNA), Universidad Estatal a Distancia (UNED) y el Instituto Tecnológico (TEC). Se presentaron varias ponencias, entre las que se pueden destacar algunos de los aportes.

Don Roberto Murillo, filósofo y entonces profesor de la Universidad de Costa Rica, reflexionó sobre la asignación de créditos a los cursos que de acuerdo con su experiencia "Se usa como referente a las horas lectivas y no al tiempo invertido en el aprendizaje" (CONARE, 1988, p. 7). Además agregó que el "cómputo de créditos debe evolucionar, para que permita valorar las realizaciones del estudiante, no con rigidez, pero si con claridad y flexibilidad" (CONARE, 1988, p. 7).

Coincide con él, el Dr. José Alberto Soto, también filósofo de la Universidad de Costa Rica, quien agrega que hasta ese momento "la asignación de créditos se ha hecho arbitrariamente" (CONARE, 1988, p. 9).

Muchos de los participantes coinciden con ambos filósofos, al observar que los créditos se asignan arbitrariamente y que esto se hace así para cumplir con las especificaciones salidas del documento de CONARE, y van más allá de los dos educadores antes mencionados. En la ponencia hecha por el Instituto Tecnológico, se llega a dos conclusiones:

- La aplicación de los criterios del sistema de asignación de créditos es subjetiva, lo cual conlleva a diferencias en el nivel de exigencia de las diferentes carreras de la 
Institución, y mucho más se marcan estas diferencias entre las instituciones de Educación Superior en carreras afines.

- La asignación de créditos conlleva a ...fuentes de problemas: La aplicación de una norma para efectos administrativos, lo cual obvia, generalmente, variables importantes del currículo (características de la disciplina, características de los estudiantes, etc. (CONARE, 1988, p. 13)

Se observan en estas intervenciones varios problemas asociado a la forma de asignar créditos, el primero de ellos la manera de asignar. Por su lado Soto se refirió al sistema como arbitrario y por otro, la ponencia del TEC que lo califica de subjetivo. El otro problema que se desprende de estas afirmaciones es el uso que se le está dando al crédito que se considera más como un indicador administrativo que como uno académico.

Aunado a lo anterior, la ponencia del Instituto Tecnológico se queja de que el crédito no proporciona, ni a los estudiantes ni a profesores "ideas de la profundidad y complejidad de una asignatura" (CONARE, 1988, p. 14). A partir del número de créditos asignado a un curso, en ese momento, 1988, no era posible determinar

el trabajo del estudiante en función de la profundidad y complejidad de los cursos: estas son variables complejas, difíciles de operacionalizar y controlar en el proceso de enseñanza-aprendizaje. (CONARE, 1988, p. 14)

Para que los créditos reflejaran esta realidad, o sea el trabajo real de los estudiantes, los funcionarios del TEC opinan que "implicaría la existencia de planes de estudio sumamente elevados en créditos" (CONARE, 1988, p. 14), pues cada curso tendría más créditos de los asignados por la dificultad de estos y la cantidad de trabajo de los estudiantes. Esto último no estaría en armonía con la política antes mencionada, aprobada por CONARE.

De acuerdo con Ana Tristán, representante de la UNED en el Seminario, el tema del crédito es un tema que provoca discusión porque se trata de homogenizar a todos los estudiantes, asignándoles un tiempo igual para desarrollar sus actividades académicas.

Además se refiere que este sistema no está ideado para ser aplicado a una universidad como la UNED, donde no hay clases presenciales y no se pueden medir los 
cursos con la definición que fue emitida en 1977 por el CONARE. Esta definición fue pensada para cursos de modalidad presencial y nunca para ser aplicada a cursos de modalidad a distancia, donde el contacto profesor alumno es casi inexistente. En esta ponencia, Ana Tristán explicó que por esta modalidad, la UNED buscó y puso en ejecución otro concepto de crédito, distinto del usado por las demás universidades estatales. Este dice: "Se atribuirá un crédito por cada 50 horas de tiempo promedio estimado como necesario para que el estudiante adquiera los conocimientos y destrezas necesarios para aprobar el curso o actividad respectiva" (Junta Universitaria.1977. Citado por CONARE, 1988, p.19).

Revisando las ponencias de otras universidades, se encontró que no solo la UNED varió su concepto de crédito. Algunas facultades de la UNA también lo hicieron, tal es el caso de la Facultad de Ciencias de la Tierra y el Mar que habla de una hora cincuenta de trabajo semanal , la de Ciencias Sociales que requiere una hora I y la de Ciencias de Salud que requiere de dos horas. Ello difiere a las tres horas reloj de trabajo semanal, pedidas en la definición de CONARE.

De acuerdo con Manuel Monestel, funcionario de la UNED, estas diferencias se dan porque

Las horas de clase que se asignan, los programas de estudio que se diseñan, los periodos que se fijan y los grados académicos que se ofrecen en nuestros centros de educación, están dados en forma muy subjetiva y casi nunca tiene en cuenta las características psicológicas del elemento humano que debe cumplir estos derroteros. (CONARE, 1988, p. 35).

Este aspecto humano que recalca Monestel, es retomado en otra ponencia por Ana Cecilia Zúñiga de la UCR, cuando hace referencia a la época en que fue concebido el crédito tal y como se formuló en CONARE, así como a la organización curricular y a la idea de estudiante que se tenía.

Dice Zúñiga que en los años setenta la idea del campo curricular era otra, entonces se pensaba que el planeamiento y organización de los cursos era un hecho aislado, cada curso se planeaba en forma individual sin tomar en cuenta nada más que las ideas e intereses del 
profesor, aunque formara parte de un todo que era la carrera, por eso a veces las carreras parecían estar un poco desarticuladas. Zúñiga explica que

El concepto de crédito se da entonces dentro de una realidad histórica, para un momento dado y de una manera estática, sin considerar las concepciones epistemológicas de las diferentes disciplinas.

Se constituye además, como una norma impuesta con la que hay que cumplir, sin que medie una participación activa de quienes están relacionados directamente con él, a saber estudiantes y profesores. (CONARE, 1988, p. 58).

Esta concepción cambia, a partir de 1980, cuando la profesora Alicia Gurdián, de la UCR, escribe Modelo Metodológico de Diseño Curricular en la que se propone una nueva conceptualización de lo que es currículo. En este texto se le da gran importancia al contexto social en el que se encuentra inmerso el plan de estudios; se toma en cuenta el mercado laboral, la definición profesional, las necesidades y expectativas de los estudiantes y profesores, la filosofía de la Universidad y los referentes teóricos y prácticos de las diferentes disciplinas. Además se le da participación activa a los recursos humanos que tienen relación con la planificación y desarrollo de los cursos.

Para Zúñiga esta forma de acercarse al currículo que fue adoptada por las universidades estatales tiene relación con la normativa de CONARE, acerca de la asignación de créditos, puesto que como se había planteado esta asignación:

Distorsiona la función del diseño y la planificación curricular, ya que en muchas oportunidades para cumplir con su definición se pierde la coherencia y secuencia de la organización de contenidos propios de una disciplina

No permite establecer una relación de importancia entre los cursos de acuerdo con su contenido temático por ser demasiado rígido e inflexible y tener que adoptarlo como tal, provoca que la adjudicación de créditos no sea producto de un proceso de análisis que oriente a valorar las características propias del curso así como su relación con otros cursos, dándose esta adjudicación en forma anárquica. (CONARE, 1988, p. 59)

Zúñiga concluye que al haberse concebido la definición de crédito en un marco histórico ya superado, al haber cambiado la forma de elaborar el currículo, donde se toman en cuenta las posiciones de los alumnos y profesores, propone que las universidades públicas analicen la operancia de este concepto así como sus implicaciones. 
Una vez recopiladas algunas de las ideas que se expusieron en este Seminario, es interesante revisar algunas de las conclusiones a que se llegó. Entre las cinco conclusiones que se presentan en el informe final del Seminario, se destaca la primera que acoge las ideas de la ponencia de Ana Cecilia Zúñiga, en el sentido de recordar que la definición de crédito adoptada en 1975, responde a una realidad histórica que quince años después, ha variado desde el punto de vista de diseño, desarrollo y evaluación curricular.

Otra de las conclusiones que recoge el documento es que pareciera existir un desconocimiento por parte de quienes asignan los créditos de la normativa que circunda el uso del crédito, así como la relación con los diferentes criterios que conforman el plan del estudio (tiempo, tipo de estudiantes, calidad y profundidad del contenido, entre otros)

Finalmente, cabe destacar, la conclusión que se refiere a los otros usos que se le han dado al crédito dentro del ámbito universitario, pues este concepto también está asociado al cobro de matrícula, a la carga docente y a las escalas salariales. El documente observa que estas otras funciones generan "prácticas inconvenientes y distorsionadas en la administración del currículo" (CONARE, 1988, p. 67).

\section{Otras funciones que cumple el crédito en la Universidad de Costa Rica}

Como se anotó en esta última conclusión, el crédito ha adquirido funciones que van más allá de las académicas. La Universidad de Costa Rica no es la excepción y este se encuentra inmerso en el proceso de planificación y desarrollo institucional, por lo que el crédito tiene implicaciones diversas. Por una parte contribuye al análisis de los costos de operación en relación con las distintas carreras que ofrece la Universidad y, por otra parte, permite determinar el logro mínimo y el rendimiento académico de los estudiantes; parámetros que son tomados en cuenta en el otorgamiento de becas y en el ingreso a carreras de cupo restringido.

En las indagaciones acerca de las implicaciones del crédito en la Universidad de Costa Rica, se puede identificar que el crédito tiene tres funciones: académico, financiero y administrativo.

Desde el punto de vista académico, el crédito se concibe como la unidad valorativa del esfuerzo estudiantil dentro y fuera del aula, durante un ciclo lectivo. La valoración involucra 
tanto el aspecto cuantitativo como el cualitativo. El aspecto cuantitativo se refleja en la medición del trabajo del estudiante dentro del aula, mediante una calificación que puede oscilar entre cinco y diez, siendo el siete la nota mínima del logro del aprendizaje en el curso. La nota de cinco equivalía a la nota de perdido o "no se presentó", actualmente este concepto ha cambiado.

En el nivel macro este aspecto se determina por medio de dos variables: "logro mínimo" y "rendimiento académico". (OPLAU, 1991). El logro mínimo es la multiplicación de los créditos matriculados por 7 , que es la nota mínima con que se aprueba un crédito (OPLAU, 1992). Para determinar el rendimiento académico se utiliza el promedio ponderado, el que se obtiene sumando los productos de la calificación final de cada curso por sus créditos y dividiendo esa suma entre el número total de créditos de las asignaturas cursadas (OPLAU, 1992).

En el aspecto cualitativo se refiere al esfuerzo intelectual que realiza el estudiante fuera del aula, para el logro de los aprendizajes y de esa manera aprobar el curso. Se parte de la premisa de que este aspecto no se toma en cuenta en la asignación de créditos a los cursos, de acuerdo con los docentes entrevistados, por ser variables difíciles de valorar y cuantificar. Razón por la cual, la valoración del trabajo del estudiante que se realiza actualmente no se puede considerar pertinente; es decir, que la relación de los créditos aprobados y las notas de los cursos no determinan un rendimiento académico real, dado que no existe una concordancia entre el número de créditos asignados y el esfuerzo fuera del aula del trabajo estudiantil.

En relación con la valoración del esfuerzo extra clase se realiza un sondeo general, para lo cual se utiliza la técnica de la entrevista abierta, con 15 profesores y 16 estudiantes de las distintas áreas del saber. En el Área de la Salud, se indaga en las Facultades de Medicina Microbiología; en el Área de Ciencias Sociales: en la Escuela de Formación Docente, Antropología, Administración Pública y Psicología; en el Área de Ciencias Básicas: en la Escuela de Física y Matemática; en el Área de Artes y Letras en las carreras de Filología, Artes Gráficas y Artes Musicales. En el Área de Ingeniería y Arquitectura en las carreras de Ingeniería Agrícola, Arquitectura y Agronomía. 
De dichas entrevistas exploratorias, se obtienen algunos indicadores que permiten determinar que el esfuerzo del trabajo intelectual estudiantil se valora desde dos perspectivas: la función docente y la función estudiantil.

Dentro de la función docente se pueden destacar algunos indicadores tales como:

$\checkmark$ El tipo de curso, distinguiéndose diferentes nomenclaturas como curso teórico, práctico, de laboratorio y otro tipo. El Centro de Evaluación Docente (CEA), clasifica los cursos como: teórico, práctico, laboratorio, taller, seminario, módulo, giras, práctica profesional y práctica clínica (Vargas y Calderón, 2004).

$\checkmark$ El nivel del curso en el plan de estudios.

$\checkmark$ La condición de curso; es decir, si es un curso específico de la disciplina, si es complementario, de servicio o de otro tipo.

$\checkmark$ Las técnicas de enseñanza docente, dado que algunos señalan que la metodología participativa, requiere mayor preparación de las actividades y mayor profundidad en el desarrollo de los temas.

Dentro de la función estudiantil se destacan indicadores tales como:

$\checkmark$ El tipo de material de estudio, si este es suficiente, pertinente y actualizado y con qué facilidad se puede adquirir.

$\checkmark$ La cantidad de material por estudiar en un determinado tiempo.

$\checkmark$ Las exigencias docentes en los exámenes y en las asignaciones, las cuales según la mayoría de las opiniones estudiantiles, no son acordes con lo que se explica o exige dentro del aula.

$\checkmark$ El trabajo que el estudiante realiza durante las horas de clase presencial o virtual.

$\checkmark$ El esfuerzo intelectual que realiza para aprobar un curso.

Desde la perspectiva financiera, el crédito se puede concebir como una unidad base que determina el monto que la Universidad de Costa Rica debe cobrar por concepto de matricula. En los archivos de la Universidad, se pueden consultar las estadísticas en cuanto a los gastos directos y totales por crédito matriculado y por crédito aprobado.

Desde la función administrativa, el crédito se puede concebir como la unidad base que determina el grado académico que otorga la Universidad de Costa Rica. Actualmente se distinguen tres niveles: el pregado que contempla el diplomado y el profesorado, el grado 
que incluye el bachillerato y la licenciatura y el posgrado con la maestría y el doctorado (CONARE, 1991)

Como se observa, el crédito ha adquirido, a lo largo de los años, otras funciones que van más allá de lo académico, y a pesar de las recomendaciones que se hicieron en el marco del Seminario de Asignación de Créditos, en el año de 1988, no se hizo ninguna revisión como lo recomendaban las conclusiones. Por esta razón, Ana Cecilia Zúñiga, en el marco del V Congreso Universitario, celebrado en 1990, presentó su ponencia, en la que recomendó un estudio del concepto de crédito, su propuesta dice

Se propone que:

- La Universidad de Costa Rica se aboque a un análisis profundo en relación con la operancia del concepto de crédito y sus implicaciones.

- Ese análisis sea producto de la participación de las diferentes instancias que conforman a nuestra universidad.

- Los resultados del análisis deben ser presentados y discutidos ante las autoridades universitarias, quienes a su vez las someterán al criterio de CONARE

- Si los resultados del análisis resultan ser divergentes con respecto a las otras universidades; es prudente que se respete el marco referencial de cada una y buscar posteriormente mecanismos que logren equiparar la conceptualización del crédito con el fin de coordinar lo referente al reconocimiento $y$ equiparación de estudios, grados y títulos así como a la transferencia de estudiantes de una a otra universidad. (Zúñiga, 1990, p. 118)

Esta propuesta fue acogida por el Quinto Congreso y este emitió la resolución, número quince, en la que se proponía que la Universidad de Costa Rica empezaría "un análisis profundo en relación con la operancia del concepto de crédito y sus implicaciones" (Quinto Congreso,1990, p. 14).

A pesar de esta resolución, emitida en el año de 1990, aún no se ha hecho este análisis profundo de la operancia del crédito. Es por esta razón, así como por los resultados obtenidos en el Seminario de Asignación de Créditos de 1988, que se ha inscrito un proyecto en el Instituto de Investigación en Educación (INIE) titulado: "Hacia la redefinición del crédito: 
una unidad valorativa de la carga académica del estudiante en la educación superior" ( $N^{\circ}$ 724-A2-172) que se propone diagnosticar el concepto de crédito y sus implicaciones en la planificación curricular y en el esfuerzo del trabajo estudiantil. En el marco del proyecto se pretende elaborar una propuesta con la finalidad de lograr una redefinición del concepto de crédito académico y establecer los mecanismos que logren equiparar este nuevo concepto de crédito en las universidades estatales.

Algunas de las razones que justifican la necesidad de plantear este tipo de investigación son:

$\checkmark$ Las unidades académicas de la Universidad de Costa Rica, constantemente están realizando reformas parciales o totales en los planes de estudio y creando nuevas carreras en las distintos pregrados, grados y posgrados. La planificación curricular incluye aspectos tales como: elaboración de perfiles profesionales, definición de objetivos de la carrera, estructura de los cursos en el plan de estudios, asignación de créditos a los cursos, relación de los cursos requisito y cursos correquisitos, entre otros aspectos. Aunque a simple vista no se ve la relevancia del crédito en la planificación curricular de una carrera, éste tiene grandes implicaciones en el proceso de desarrollo de la carrera, dado que determina la carga académica que puede matricular un estudiante de tiempo completo, permite llevar un control de los cursos que tiene cada carrera, permite el proceso de equiparar los grados y títulos entre las universidades públicas. Así como también le facilita al estudiante organizar su carga académica de acuerdo con su disponibilidad de tiempo para el estudio.

$\checkmark$ Por otra parte, la concepción de crédito que se adopte es un factor que influye en la planificación curricular de las carreras en educación superior. Dentro de las indagaciones realizadas hasta el momento, se tiene que la interpretación de la definición de crédito varía según la institución: en la Universidad Estatal a Distancia (UNED), por ejemplo, la asignación de créditos a un curso es el resultado de la opinión de especialistas en la materia y de la opinión de estudiantes. En la Universidad de Costa Rica (UCR), no se toma en cuenta la opinión de los estudiantes, generalmente lo decide el coordinador de la carrera y algunas veces se le pide opinión a los docentes. En el Instituto Tecnológico (ITEC) y en la Universidad Nacional (UNA), el procedimiento es muy similar al de la UCR (Seminario sobre créditos CONARE, 1988). 
$\checkmark$ Actualmente el concepto de crédito se utiliza para diversos fines en la Universidad de Costa Rica lo que podría responder en una forma particular de interpretar la concepción y aplicación. Algunos de los usos más frecuentes son:

- Cobro de matrícula

- Asignación de becas de acuerdo con el mínimo y el máximo de créditos.

- Determinación del número de horas lectivas, las que generalmente coinciden con el número de horas lectivas presenciales.

- Determinación de la carga académica del estudiante, sugiriendo 18 créditos por ciclo lectivo.

- Determinación del número de créditos en los planes de estudio según el nivel académico (pregrado, grado y posgrado), lo que ocasiona, muchas veces, rigidez e inflexibilidad en los planes de estudio.

$\checkmark$ El tema del crédito académico ha despertado preocupación en el seno de las universidades estatales. Esto se refleja en el Seminario sobre créditos realizado por el CONARE en 1988, así como los acuerdos del Quinto Congreso Universitario, donde se plantea (resolución 15), la necesidad de que la Universidad de Costa Rica se aboque a un análisis profundo en relación con la operacionalización del concepto de crédito y sus implicaciones. Este análisis debe considerar tres aspectos básicos: primero que se les dé participación a las diferentes instancias de la institución; segundo que los resultados se sometan a criterio del CONARE y tercero, que se encuentren los mecanismo que logren equiparar la conceptualización de créditos en las otras universidades estatales (Propuesta de resoluciones del V Congreso Universitario, 1990). Hasta la fecha no se conocen investigaciones que aborden esta temática y cada día se hace más patente la necesidad de reconceptualizar el crédito como unidad valorativa del trabajo estudiantil. Esto porque las condiciones del estudiante de tiempo completo han variado así como las estrategias que utiliza para realizar su trabajo dentro y fuera de la clase.

$\checkmark$ Otra razón que justifica la razón de este estudio, radica en que el grupo de docentes entrevistados de distintas áreas académicas, consideran que la asignación de créditos a los cursos es un procedimiento arbitrario. Se cuestionan la relación entre el número de créditos, las horas de clases presenciales y virtuales y el trabajo estudiantil, lo que puede influir en su rendimiento académico. 


\section{El crédito en Europa: una realidad que cambia}

El tema del crédito no es solo preocupación en el ámbito nacional o de la Universidad de Costa Rica, en Europa, actualmente es una cuestión que se encuentra en discusión a raíz del proyecto Tuning.

Este es un proyecto propio de las universidades europeas que componen la Comunidad Europea, y que tiene por objetivo "contribuir significativamente a la creación del Espacio Europeo de Educación Superior" (Tunning, 2003, p. 18) para buscar consenso entre casas de educación superior, así como el respeto a las diversidades culturales y sociales, a la transparencia y a la confianza para llegar a puntos de referencia comunes. Puntos de referencia que están basados en los resultados del aprendizaje de los estudiantes universitarios, en las competencias, habilidades y destrezas, todo ello lo que busca es encontrar una guía hacia lo que se puede considerar "lo común" en el marco de la Unión Europea.

Con este proyecto también se busca una lograr mayor calidad académica en la universidad europea, y entre los puntos de interés se encuentra el tema del crédito que busca conseguir una definición para un uso común en toda Europa. Esta iniciativa tiene por objetivo permitir a los estudiantes de los países europeos trasladarse de universidades, dentro del ámbito de la Comunidad, sin perder los créditos ya ganados en la universidad de procedencia.

La idea tanto de búsqueda de una excelencia académica, así como de una equiparación de créditos surgió a partir de Declaración conjunta de los Ministros Europeos de Educación, reunidos en Bolonia el 19 de junio de 1999.

En la Declaración emitida, se enumeran los objetivos de calidad académica que buscan para las universidades los ministros de Educación, con el fin de lograr un sistema universitario europeo. Dentro de los objetivos propuestos se encuentra el siguiente, que se relaciona con el tema del crédito:

El establecimiento de un sistema de créditos - similar al sistema de ETCS - como medio adecuado para promocionar una más amplia movilidad estudiantil. Los créditos se podrán conseguir también fuera de las instituciones de educación superior, 
incluyendo la experiencia adquirida durante la vida, siempre que esté reconocida por las Universidades receptoras involucradas. (Declaración de Bolonia, 1999)

Se observa que el concepto de crédito aquí referido, va más allá de lo que comúnmente se ha conocido, puesto que un estudiante tiene la posibilidad de ganar créditos fuera de las instituciones de la educación superior e incluso, puede ganarlos en la experiencia que le otorga la vida. Eso sí, es importante tomar en cuenta que estas opciones, mencionadas anteriormente, deberán estar registradas y avaladas por las respectivas universidades

El crédito en Europa se concibe desde tres perspectivas: como la carga académica del estudiante, los resultados de aprendizaje y las horas de contacto en el aula. Tal y como se enuncia en la siguiente apreciación.

Un sistema de créditos es una forma sistemática de describir un programa de educación asignando créditos a sus componentes. La definición de los créditos en los sistemas de educación superior puede basarse en distintos parámetros, como la carga de trabajo del estudiante, los resultados del aprendizaje y las horas de contacto. (Programa Erasmus, 2005)

Los países miembros de la Unión Europea consideraron que 60 créditos miden la carga de trabajo de un estudiante a tiempo completo durante un curso académico. La carga de trabajo para un estudiante en un programa de estudios a tiempo completo equivale, en la mayoría de los casos, entre treinta y seis y cuarenta semanas por año, y en tales casos un crédito representa de 24 a 30 horas de trabajo. La carga de trabajo se refiere al tiempo teórico en que se puede esperar que un estudiante obtenga los resultados del aprendizaje requeridos. (Proyecto Erasmus, 2005)

El crédito en Europa, es también una forma de cuantificar los resultados del aprendizaje. Los resultados del aprendizaje son conjuntos de competencias que expresan lo que el estudiante sabrá, comprenderá o será capaz de hacer tras completar un proceso de aprendizaje, corto o largo.

La declaración de Bolonia hace referencia al ECTS, este es el Sistema Europeo de Transferencia y Acumulación de Créditos, que es pertinente mencionar pues se considera la 
institución oficial encargada de transferir los créditos ganados por un alumno de una universidad a otra

Lo que busca este Sistema de Transferencia es que los estudiantes tengan la posibilidad de que diferentes universidades de la Comunidad Europea, reconozcan sus estudios y la carga académica que poseen en el caso retrasladarse de universidad.

Este Sistema de Transferencia se basa en la carga académica del estudiante,

El Sistema europeo de transferencia y acumulación de créditos es un sistema centrado en el estudiante, que se basa en la carga de trabajo del estudiante, necesaria para la consecución de los objetivos de un programa. Estos objetivos se especifican preferiblemente en términos de los resultados del aprendizaje y de las competencias que se han de adquirir. (Proyecto Erasmus, 2005)

Este sistema de transferencia está inscrito en el Proyecto Erasmus, este último es el encargado de la cooperación y movilidad en el ámbito de la enseñanza superior europea, y su objetivo es "realzar la calidad de la enseñanza superior europea y favorecer la comprensión intercultural gracias a la cooperación con los terceros países" (Proyecto Erasmus, 2005).

El programa permite además, a los estudiantes y a los docentes universitarios de todo el mundo cursar estudios de posgrado en las universidades europeas, y fomentar, al mismo tiempo, la movilidad de los estudiantes y universitarios europeos hacia terceros países, sin problema en el reconocimiento de de carga académica de créditos.

El sistema de transferencia ha logrado una gran movilidad de los estudiantes en Europa, estimulando que la educación de éstos sea mejor pues el tener contacto con varias universidades amplia su conocimiento.

EI ECTS es el único sistema de créditos que se ha ensayado y utilizado con éxito en toda Europa. EI ECTS se estableció inicialmente para la transferencia de créditos: el sistema facilitaba el reconocimiento de los períodos de estudios en el extranjero, incrementando así la calidad y el volumen de la movilidad de los estudiantes en Europa. EI ECTS se está convirtiendo ahora en un sistema de acumulación que podrá 
aplicarse a nivel institucional, regional, nacional y europeo. Éste es uno de los objetivos clave de la declaración de Bolonia de junio de 1999. (Proyecto Erasmus, 2005)

La asignación de créditos ECTS se basa en la duración oficial de un ciclo de estudios. La carga de trabajo total necesaria para obtener un título de primer ciclo que dure oficialmente de tres o cuatro años, se expresa como 180 ó 240 créditos. La carga de trabajo del estudiante en el ECTS, incluye el tiempo invertido en asistencia a clases, seminarios, estudio personal, preparación y realización de exámenes.

Se asignan créditos a todos los componentes educativos de un programa de estudios (como módulos, cursos, períodos de prácticas, trabajos de tesis, etc.). Estos reflejan el volumen de trabajo que cada componente requiere en relación con el volumen total de trabajo necesario para completar un curso entero de estudio en el programa elegido.

La asignación de créditos ECTS permite a los centros traducir sus estructuras académicas a una unidad de medida común, sin que ello suponga modificar las estructuras existentes. En ciertos países, la asignación de créditos ECTS se reduce a una simple operación aritmética, mientras que, en otros, requiere revisión de los programas de estudio con negociaciones a nivel de facultad, departamento o centro.

La flexibilidad es un aspecto esencial de la filosofía del ECTS, especialmente por lo que se refiere a la asignación de créditos. Deben asignarse créditos ECTS a todas las unidades de curso impartidas - ya se trate de cursos obligatorios o facultativos - así como a los trabajos prácticos, proyectos de final de estudios y periodos de prácticas en empresas, a condición de que estas "unidades" formen parte del programa de estudios oficial, incluidos los estudios de postgrado, y que los trabajos del estudiante sean objeto de una evaluación. Los créditos ECTS no se basan en las horas de asistencia en sí mismas, sino más bien en el volumen total de trabajo que éstas implican.

España ha sido uno de los países europeos, perteneciente a la Unión Europea, que aún no ha adoptado el concepto de crédito y por ende tampoco se han adscrito al ECTS. Actualmente este país tiene una noción diferente del crédito que no está acorde con la normativa adoptada por la comunidad europea, tal y como se estructuró a partir de la declaración de Bolonia, en el Proyecto Tuning. 
La legislación española actual define las unidades de crédito como unidades de acumulación, unidades que tienen en cuenta las horas lectivas (clases teóricas y/o prácticas) pero en ningún caso, se tiene en cuenta el trabajo del estudiante.

Por lo anterior, si España se adapta a la legislación europea deberá tener en cuenta que el crédito tiene que estar basado en el trabajo que el alumno realiza para tener la formación adecuada (conocimientos y competencias). Asimismo se obliga a tener en cuenta lo que el alumno necesita saber y va a depender del contenido de las clases teóricas, clases prácticas, seminarios, tutorías evaluaciones, entre otras. También se considera que es necesario observar el trabajo del profesor, pues este es relevante para ayudar al alumno en su formación.

Finalmente, para que España se adapte a la normativa europea, el concepto de unidad de crédito debe reflejar el trabajo necesario para lograr una formación académica integral mediante un aprendizaje adecuado que permita desarrollar en el alumno una capacidad de análisis.

Una vez que se adopten las medidas antes mencionadas, el crédito español, podría definirse como:

(...) unidad de valoración de la actividad académica que integra armónicamente las enseñanzas teóricas y prácticas, otras actividades académicas dirigidas y el trabajo personal del estudiante, permitiendo así medir el volumen total de trabajo que el estudiante debe realizar para superar cada una de las asignaturas. (Comunidad Europea, 2005)

De esta manera, los europeos, mediante diversos programas, proyectos y declaraciones, están trabajando no solo en formular la noción de crédito, sino también en hacerla semejante en todos los países que componen la Unión Europea. Esto con el fin de facilitar el intercambio de estudiantes entre las distintas universidad, para que todos tengan no solo una formación similar, sino un volumen de trabajo parecido. Todo ello va encaminado a buscar una excelencia académica que sea compatible entre todas las universidades que se adhirieron a la Declaración de Bolonia. 


\section{Consideraciones Finales}

Como consideraciones finales del artículo se ofrecen las conclusiones más relevantes de las entrevistas abiertas realizadas con los directores y docentes de las unidades académicas de la Universidad de Costa Rica, así como a funcionarios del CONARE, que participan en el proceso de reconceptualización del crédito. Es importante señalar que esta información servirá de base para elaborar los instrumentos del diagnóstico. Entre los aspectos más relevantes se pueden citar:

- En los procesos de autoevaluación que siguen algunas escuelas que participan en el proyecto, la asignación de créditos ha sido un problema. Es necesario revisar los créditos, apuntan las personas entrevistas, "con el cambio en las técnicas de enseñanza y el número de estudiantes matriculados, el sistema ya no se ajusta a las necesidades de la carrera". No saben cómo aplicar la definición de crédito, de acuerdo con las particularidades y el nivel de dificultad de cada curso.

- El problema de la asignación de créditos tiene relación con el número de créditos establecido por ciclo. La preocupación tiene que ver con el número total de créditos y no con los diferentes niveles del contenido de los cursos. Se ajustan a las normativas, aunque se eliminen curso que se consideren importantes, con el propósito de no sobrepasar el número de créditos por ciclo y por grado. Los cursos de servicio se coordinan con las otras carreras para la asignación de créditos, siguiendo el mismo criterio

- El número de créditos afecta tanto a los docentes en la asignación de carga académica como a los estudiantes en la categoría de beca que se le asigna y el costo de la matrícula. Por ejemplo, en las carreras de artes la mayoría de los cursos tiene baja denominación; uno o dos créditos; el estudiante matricula 8 créditos y no le alcanza para solicitar beca. Algunas estudiantes se ven en la necesidad de interrumpir estudios porque no pueden pagar la universidad, la carrera es muy cara y no les alcanza los créditos para la beca.

- Los estudiantes tardan más años en terminar la carrera que lo establecido en los planes de estudio. En algunos casos, hasta 10 y 12 años; esto por porque los educandos abandonan la carrera por algunos ciclos lectivos o matriculan cursos muy exigentes que tienen pocos créditos. Algunos directores entrevistados, han señalado que el número de créditos no corresponde al nivel de exigencia, por lo que se produce deserción en los cursos. Indican que ante este fenómeno algunos profesores han Volumen 5, Número 2, Año 2005, ISSN 1409-4703 26 
tenido que bajar el nivel de exigencia del curso para lograr la permanencia de los estudiantes.

- La mayor parte de los cursos son de carácter teórico, práctico, o teórico-práctico. De este último se distinguen tres modalidades: teórico con horas prácticas, teórico con horas de laboratorio y teórico con horas prácticas y de horas de laboratorio. Algunos de estos cursos tienen las horas de práctica y laboratorios separadas de la teoría. En este caso el estudiante lleva dos cursos que se dan en diferentes horarios pero con único número de créditos, por ejemplo el curso "Diseño Gráfico" que tiene asignado 3 créditos: 4 horas teóricas y dos horas prácticas o de laboratorio en otro horario, un total de 6 horas presenciales. La otra modalidad es el curso que tiene incluidas las horas de teoría y práctica o laboratorio, a manera de ejemplo el curso Geografía Regional de Costa Rica" que tiene asignado 3 créditos, dos horas presenciales y una hora más para consultar en la Escuela. En estos ejemplos se observa una diferencia del doble de horas para la misma cantidad de créditos.

- Se encuentran cursos de uno, dos o tres créditos. Aunque tiene diferente creditaje, en su gran mayoría tienen 4 horas presenciales, tareas semanales, actividades en clase y prácticas fuera del aula.

- Los cursos de laboratorio están subvalorados en créditos, porque algunos son de 6 horas presenciales, más prueba corta e informe y solo tienen asignados 2 créditos.

- Otro de los problemas en el plan de estudios, es la asignación de créditos a los cursos de servicios, como por ejemplo el de Química General que tiene 3 créditos. Este presenta condiciones muy diferentes de otros cursos de servicios, dado que se ofrece en un auditorio con 160 personas por curso, no existiendo ninguna interrelación del profesor con los alumnos y los exámenes se corrigen por medio de lectora óptica. Este curso además se reproduce de la misma manera las distintas sedes de la Universidad.

- También el crédito tiene implicaciones económicas, dado que se utilizan para calcular el cobro de matrícula y algunas actividades académicas que no tienen créditos, como los cursos "Investigación Dirigida I, II y II" que corresponden a la modalidad de tesis como trabajo final de graduación y la actividad deportiva, se le asigna un creditaje únicamente para efectos de pago; al curso de "Investigación Dirigida" dos créditos y a la "Actividad Deportiva" medio crédito.

- También se ha observado que en las unidades académicas se distinguen tipos de práctica tales como: elemental, instrumental, clínico y profesional. Cada uno de estos Volumen 5, Número 2, Año 2005, ISSN 1409-4703 
tipos de práctica responde a diferentes niveles de dificultad que deberían de tomarse en cuenta en el momento de la asignación de créditos.

- En el nivel de pregrado y grado se distinguen cursos masivos de 150 estudiantes, cursos atención individual (modalidad de tutoría o cursos especializados de las artes), cursos con cupo de 20, 25, 30 o 35 estudiantes, cursos sobrecargados con 40 y 45 estudiantes que realizan matricula por inclusión. Todos estos aspectos deberían tomarse en cuenta cuando se asigne el creditaje.

Además de las anteriores consideraciones finales es importante recalcar que este trabajo presenta las ideas de un proceso que apenas se inicia. La investigación que se está desarrollando sobre el crédito es un proceso de tres etapas. La primera corresponde al diagnóstico, el cual se encuentra en la etapa de construcción y validación del documento tomando en cuenta el criterio de directores de Unidades Académicas, docentes, estudiantes y expertos de CONARE. Una vez concluido este proceso, se procederá a aplicarlo en diferentes Unidades Académicas de la Universidad de Costa Rica, para obtener datos del diagnóstico acerca de la situación del crédito.

Una segunda etapa será la elaboración de una propuesta de cálculo de crédito y la aplicación de la misma en algunas Unidades Académicas, como un plan piloto. Para la tercera etapa se propone un proceso de divulgación y asesoría de la "nueva concepción de crédito" en las otras instituciones de educación pública superior del país.

\section{Referencias}

Comunidad Europea. (2005). Education and Training. Recuperado el 24 de octubre de 2005, de http://europa.eu.int/comm/education/programmes/socrates/socrates en.html.

Comunidad Europea. (2005). Programa Erasmus. En: Education and Training. $\begin{array}{llllll}\text { Recuperado el } & 14 & \text { de } & \text { julio, } & \\ \end{array}$ https://habidis.cpd.um.es:8020/servlet/um.erasmus.ControlErasmus?opcion=menu, http://europa.eu.int/comm/education/programmes/socrates/tnp/applicant es.html

CONARE. (1977). Nomenclatura de grados y títulos en la educación superior. San José, Costa Rica: Documento mimiegrafiado.

CONARE. (1982). Guía para la presentación de planes de estudio. Elaborada por los asesores de la Vicerrectoría de Docencia, San José Costa Rica: Documento mimiegrafiado. 
CONARE. (1988). Seminario Asignación de créditos en la Educación Superior. Informe final. San José, Costa Rica: Documento mimiegrafiado.

CONARE. (1991). Grados académicos en la educación superior. San José, Costa Rica: Documento mimiegrafiado.

Ministros de Educación europeos, Declaración de Bolonia. (1999). El espacio europeo de la educación superior. Recuperado el 7 de julio 2005, de http://www.aneca.es/modal eval/docs/declaracion bolonia.pdf

Martín, Minor. (1988). Informe final de Asignación de Créditos de la Educación Superior. San José, Costa Rica: CONARE.

Tuning Educational Structures in Europe. (2003). Informe final. Proyecto Piloto-Fase 1. Bilbao: Universidad de Deusto.

Universidad de Costa Rica, Consejo Universitario. (2002). Estatuto Orgánico. Recuperado el 28 de julio del 2005, de http://cu.ucr.ac.cr/

Universidad de Costa Rica. (2001). Facultades y Escuelas. Recuperado el 28 de julio del 2005, de http://www.ucr.ac.cr/descripcion/areas.htm

Universidad de Costa Rica, Consejo Universitario. (2001). Reglamento de Régimen Académico Estudiantil. San Pedro, Costa Rica: Oficina de Publicaciones.

Universidad de Costa Rica, OPLAU. (1992). Gastos unitarios de docencia 1989-1990. San Pedro, Costa Rica: Oficina de Publicaciones.

Universidad de Costa Rica, OPLAU. (1991). Gastos unitarios de docencia 1985-1988. San Pedro, Costa Rica: Oficina de Publicaciones.

Universidad de Costa Rica. (1990). Estatuto Orgánico. San Pedro, Costa Rica: Oficina de Publicaciones

Universidad de Costa Rica. (1990). Quinto Congreso Universitario, resolución № 15. San Pedro, Costa Rica: Oficina de Publicaciones.

Universidad de Costa Rica, Consejo Universitario. (1976). Sesión 2297. San Pedro, Costa Rica: Oficina de Publicaciones.

Universidad de Costa Rica. (1968). Estatuto Orgánico. San Pedro, Costa Rica: Oficina de Publicaciones.

Universidad de Costa Rica. (1962). Estatuto Orgánico. San Pedro, Costa Rica: Oficina de Publicaciones.

Universidad de Costa Rica. (1956). Estatuto Orgánico. San Pedro, Costa Rica: Oficina de Publicaciones.

Universidad de Costa Rica, Consejo Universitario. (1953). Acta No 80. San Pedro, Costa Rica: Oficina de Publicaciones. 
Universidad de Costa Rica, Consejo Universitario. (1952). Acta N 50. San Pedro, Costa Rica: Oficina de Publicaciones.

Vargas, Alicia; Calderón, María Lourdes. (2004). Sistema de evaluación docente en la Universidad de Costa Rica. San Pedro, Costa Rica: Universidad de Costa Rica, Centro de Evaluación Académica.

Zúñiga, Ana Cecilia. (1990). El crédito como unidad valorativa de la Universidad de Costa Rica. Ponencia para el Seminario de Asignación de créditos en la Educación Superior. En: V Congreso Universidad de Costa Rica. Comisión Excelencia Académica. San Pedro, Costa Rica: Oficina de Publicaciones Universidad de Costa Rica. 